Series A

I. MATHEMATICA

$336 / 3$

\title{
ITERATION DER REELLEN POLYNOME ZWEITEN GRADES III
}

VON

P. J. MYRBERG 
Vorgelegt am 14. Dezember 1962. 


\section{Einleitung}

1. Zweck der vorliegenden Arbeit ist es, neue Beiträge zur Iterationstheorie der reellen quadratischen Polynome zu liefern. Indem wir uns im folgenden auf die reellen Werte der Variablen einschränken, ist unsere Absicht, ein allgemeines Verfahren zur Bildung von attraktiven Zyklen des gegebenen Polynoms darzustellen. Unsere Methode wird auf die Bestimmung von Nullzyklen des Polynomes begründet, also Zyklen, deren Multiplikator gleich null ist und deren Punkte somit stark attraktive Fixpunkte für das zugeordnete iterierte Polynom sind.

Es sei

$$
y_{1}=y^{2}-p
$$

das gegebene Polynom, wo $p$ ein reeller Parameter ist. Durch $n$-fache Iteration von $(1,1)$ gelangt man zu einem Polynom

$$
y_{n}=P_{n}(y)
$$

vom Grade $2^{n}$, dessen Koeffizienten ganze rationale Funktionen von $p$ sind. Insbecondere ist der letzte Koeffizient

$$
P_{n}(0)=G_{n}(p)
$$

ein Polynom von $p$ vom Grade $2^{n-1}$. Die ersten unter diesen Polynomen sind

$$
G_{1}(p)=-p, \quad G_{2}(p)=p^{2}-p, \quad G_{3}(p)=\left(p^{2}-p\right)^{2}-p,
$$

und sie können vermittels der Rekursionsformel

$$
G_{n+1}(p)=G_{n}^{2}(p)-p
$$

berechnet werden.

2. Es sei nun $y_{0}$ ein endlicher Fixpunkt von $(1,2)$ und

$$
y_{1}, y_{2}, \ldots, y_{n-1}, y_{n}=y_{0}
$$

die daraus vermittels der Iteration von $(1,1)$ erhaltenen Bildpunkte, welche bei Anwendung von $(1,1)$ miteinander zyklisch permutiert werden. Aus dem Ausdruck 


$$
S=P_{n}^{\prime}\left(y_{v}\right)=2^{n} y_{0} y_{1} \ldots y_{n-1}
$$

des Multiplikators des von den Punkten $(2,1)$ gebildeten Zyklus $\Sigma$ geht hervor, dass $\Sigma$ attraktiv ist, wenn

$$
\left|y_{0} y_{1} \ldots y_{n-1}\right|<\frac{1}{2^{n}} \text {. }
$$

Ist speziell $y_{0}=0$, so hat man es mit einem Nullzyklus zu tun. Ihre Punkte bestehen aus

$$
-p, p_{1}, p_{2}, \ldots, p_{n-2}, p_{n-1}=0
$$

wo $p_{v}=G_{v+1}(p)$ und

$$
G_{n}(p)=0
$$

Wie wir schon früher bewiesen haben [3], können attraktive Zyklen nur für die Parameterwerte des Intervalles

$$
\frac{1}{4}<p<2
$$

vorkommen. Aus einem allgemeinen Satz von FATou folgt, dass es höchstens einen attraktiven Zyklus gibt und dass ihre Punkte zum Intervall

$$
-q_{1}<y<q_{1}
$$

gehören, wo

$$
q_{1}=\frac{1}{2}-\sqrt{\frac{1}{4}+p}
$$

ein repulsiver Fixpunkt ron $(1,1)$ ist.

Bei der Bestimmung der attraktiven Zyklen spielen die reellen Nullstellen der Polynome $G_{n}(p)$ eine wichtige Rolle. Denn ist $p_{0}$ eine solche Nullstelle, so gibt es, wegen der Stetigkeit ron $S(p)$. ein Intervall $\left|p-p_{0}\right|<\varepsilon$, für dessen $p$-Werte stets ein attraktiver Zyklus existiert.

Aus der Definition der $G$-Polrnome geht hervor, dass jede Nullstelle des Polynoms $G_{n}(p)$ zugleich eine Nullstelle sämtlicher Polynome $G_{q n}$ $(q=2,3, \ldots)$ ist. Insbesondere gilt

$$
G_{n}(0)=0, \quad G_{2 n}(1)=0,(n=1,2,3, \ldots) .
$$

Wir können uns somit auf die primitiven Nullstellen von $G_{n}(p)$ einschränken, d.h. Nullstellen $p_{0}$, für welche $G_{m}\left(p_{0}\right) \neq 0$ für $m<n$ ist. 


\section{Darstellung der reellen Nullstellen des Polynoms $G_{n}(p)$ dureh Radikale}

3. Aus der Gleichung:

$$
G_{n}(p)=0
$$

ergibt sich zunächst formal für ihre Lösungen die Darstellung

$$
p=\sqrt{p \pm \sqrt{p \pm \cdots \pm V_{n-2} p}}
$$

als Radikalgleichung. Es ist eine für das Folgende zentrale Frage, welche unter den Gleichungen $(3,2)$ reelle Lösungen der Gleichnng (3.1) liefern.

Als einfachste Beispiele solcher Gleichungen geben wr die für $n=3$ erhaltenen zweigliedrigen Gleichungen

$$
p=\sqrt{p+\sqrt{p}} . \quad p=\sqrt{p-\sqrt{p}} .
$$

von denen nur die erste eine nichttriviale Nullstelle $(p \neq 0,1)$ des Polynoms $G_{3}(p)$ liefert, die den approximativen Wert

$$
p \approx 1,7548776662
$$

hat. Unter den dreigliedrigen Gleichungen

$$
\begin{array}{ll}
p=\sqrt{p+\sqrt{p+\sqrt{p}},} \quad p=\sqrt{p+\sqrt{p-\sqrt{p}}} \\
p=\sqrt{p-\sqrt{p+\sqrt{p}},} \quad p=\sqrt{p-\sqrt{p-\sqrt{p}}}
\end{array}
$$

haben nur die beiden oberen nichttriviale Lösungen, nämlich

$$
p \approx 1,9407998065, \quad p \approx 1.3107026413,
$$

welche also die einzigen primitiven Nullstellen des Polynoms $G_{4}(p)$ liefern.

4. Indem wir jetzt zur allgemeinen Lösung unserer Aufgabe übergehen, schreiben wir den rechts in $(3,2)$ auftretenden Wurzelausdruck in der symbolischen Form

$$
r=m_{1}^{+} n_{1}^{-} m_{2}^{+} n_{2}^{-} \ldots m_{q}^{+} n_{q}^{-},
$$

wenn die Folge der Vorzeichen der Radikale gleich

$$
+^{m_{1}}{ }^{n_{1}}+{ }^{m_{2}}-{ }^{n_{2}} \ldots+{ }^{m_{q}}-{ }^{n_{q}}
$$

ist. 
Es seien nun $r$ und

$$
\tilde{r}=\tilde{m}_{1}^{+} \tilde{n}_{1}^{-} \tilde{m}_{2}^{+} \tilde{n}_{2}^{-} \ldots \tilde{m}_{q}^{+} \tilde{n}_{q}^{-}
$$

zwei Radikale gleicher Ordnung

$$
N=\sum_{\nu=1}^{q}\left(m_{v}^{+}+n_{\nu}^{-}\right)=\sum_{\nu+1}^{q}\left(\tilde{m}_{v}^{+}+\tilde{n}_{\nu}^{-}\right),
$$

die also durch Nullsetzen des Polynoms $G_{N+2}$ entstehen. Unter der Annahme, dass die beiden Radikale $r$ und $\tilde{r}$ für einen gegebenen reellen Wert $p$ reell sind, gilt die Ungleichung $\tilde{r}>r$ unter den folgenden Bedingungen, wie leicht bestätigt werden kann:

Erstens wenn $\tilde{m}_{1}>m_{1}$. Zweitens wenn $\tilde{m}_{1}=m_{1}$ und gleichzeitig $\tilde{n}_{1}<n_{1}$ bei geradem $\tilde{n}_{1}$, dagegen $\tilde{n}_{1}>n_{1}$ bei ungeradem $n_{1}$ usw. Allgemein bestätigt man, dass im Falle $\tilde{m}_{1}=m_{1}, \tilde{n}_{1}=n_{1}$ die Ungleichung $\tilde{r}>r$ bei ungeradem $n_{1}$ besteht, wenn

$$
\tilde{r}_{1}=\left(\tilde{m}_{2}^{+} \tilde{n}_{2}^{-} \ldots \tilde{m}_{q}^{+} n_{q}^{-}\right)<r_{1}=\left(m_{2}^{+} n_{2}^{-} \ldots m_{q}^{+} n_{q}^{-}\right),
$$

und bei geradem $n_{1}$, wenn $\tilde{r}_{1}>r_{1}$. Durch diese rekursive Regel kann man zwei beliebige reelle Radikale auch verschiedener Ordnung miteinander vergleichen.

Nach diesen Vorbereitungen betrachten wir die Radikalgleichung

$$
p=r(p)=m_{1}^{+} n_{1}^{-} \ldots m_{q}^{+} n_{q}^{-} .
$$

Für die Existenz einer reellen Lösung von $(4,3)$ ist offenbar notwendig, dass sämtliche Teilradikale

$$
r_{v}=m_{v}^{+} n_{v}^{-} \ldots m_{q}^{+} n_{q}^{-} \quad(v=2,3, \ldots, q)
$$

für den betreffenden Wert reell sind, dass also $p \geqq r_{v}$. Man hat also für die Existenz einer reellen Lösung von $(4,3)$ die notwendigen Bedingungen

$$
r \geqq r_{v}, \quad(v=2,3, \ldots, q) .
$$

Wir behaupten, dass unsere Bedingungen $(4,4)$ auch hinreichend sind.

Zum Beweis lassen wir den Parameter $p$ das Intervall $\Delta(2>p>1)$ beschreiben. Für den Anfangswert $p=2$ sind offenbar alle Radikale $r_{\text {, }}$ reell und $r<p$. In dem extremen Fall, wo $r(p)$ lauter + Zeichen enthält, ist $r(1)>1$. Aus der Stetigkeit von $r(p)$ folgt aber, dass es im Intervalle $\Delta$ stets einen und offenbar nur einen einzigen Wert $p$ gibt, für welchen $p=r(p)$. Wenn zweitens auch - Zeichen vorkommen, gelangt man schliesslich zu einem Wert $p_{0}$, für welchen ein Teilradikal $r_{v}$ gleich Null wird. Weil dann

$$
p_{0}-r\left(p_{0}\right) \leqq p_{0}-r_{v}\left(p_{0}\right)=0
$$


so ist damit bewiesen, dass die Gleichung $(4,3)$ wenigstens eine Wurzel im Intervall $\Delta$ besitzt.

Man bestätigt leicht, dass in $(4,5)$ das Gleichheitszeichen nur für imprimitive Lösungen bestehen kann.

Wir wollen unsere Resultate im folgenden Satz zusammenfassen.

Satz 1. Die Radikalgleichung

$$
p=r(p)
$$

besitzt reelle Lösungen stets und nur dann, wenn die Bedingungen

$$
r \geqq r_{v} \quad(v=2,3, \ldots, q)
$$

erfüllt sind.

Es sei $p_{0}$ eine primitive Lösung von $(4,3)$. Offenbar ist $p=p_{0}$ die einzige primitive Lösung, wenn $r^{\prime}\left(p_{0}\right)<0$, d.h. wenn

$$
p_{0}>\frac{1}{2}\left(1+\sum_{\nu=1}^{N-2} \frac{1}{2^{v} p_{1} p_{2} \ldots p_{v}}\right) .
$$

Ich habe das Bestehen dieser Bedingung in zahlreichen Fällen bestätigt. Dass sie allgemein gültig ist, ist meine Hypothese - im folgenden mit $H$ bezeichnet - , für welche es mir nicht gelungen ist, einen allgemeinen Beweis $\mathrm{zu}$ finden.

Wir werden in den folgenden Untersuchungen die Richtigkeit unserer Hypothese $H$ annehmen, um im Nachtrag diejenigen Modifikationen unserer Sätze zu geben, welche von $H$ unabhängig sind.

5. Nach dem Obigen können die Radikale bestimmter Ordnung in eine monoton wachsende Folge geordnet werden, wo das letzte Glied lauter + Zeichen hat. Wir behaupten nun, dass man von einem Glied aus zum folgenden durch Abänderung eines einzigen Zeichens gelangt.

Zum Beweis unserer Behauptung betrachten wir zwei Glieder der Folge, $r$ und $\tilde{r}>r$, mit den Entwicklungen

$$
r=(\cdots-\cdots- \pm \pm), \quad \tilde{r}=(\cdots+\cdots+ \pm \pm),
$$

wo die mit $\cdots$ und $\cdots$ bezeichneten Zeichenfolgen miteinander bzw. übereinstimmen. Es sei $\alpha$ bzw. $\beta$ die Anzahl der - Zeichen in den genannten Folgen. Nach der in Nr. 4 gegebenen Regel ist $\alpha=2 n$ eine gerade Zahl. Ist nun erstens $\beta$ ungerade, also $\beta=2 m+1$, so liegt das Radikal $n$-ter Ordnung

$$
\varrho=(\cdots-\cdots+ \pm \pm)
$$

zwischen $r$ und $\tilde{r}$, also 


$$
r<\varrho<\tilde{r}
$$

Für $\beta=2 m$ hat das Radikal

$$
\tilde{\varrho}=(\cdots+\cdots- \pm \pm()
$$

die erforderte Eigenschaft. Wir haben also in den beiden genannten Fällen zwischen den Radikalen $r$ und $\tilde{r}$ ein neues reelles Radikal eingeschaltet. Dies gilt aber anch für die übrigen drei Fälle. Man hat nämlich im Falle

$$
\begin{aligned}
& r=(\cdots+\cdots+ \pm \pm), \quad \tilde{r}=(\cdots+\cdots- \pm \pm) \\
& \varrho=(\cdots+\cdots+ \pm) \text { für } \beta=2 m \\
& \varrho=(\cdots-\cdots+ \pm \pm) \text { für } \beta=2 m-1 .
\end{aligned}
$$

im Falle

$$
\begin{array}{ll}
r=(\cdots+\cdots- \pm \pm) . & \tilde{r}=(\cdots-\cdots- \pm) \\
\varrho=(\cdots-\cdots- \pm \pm & \text { für } \beta=2 n \\
\varrho=(\cdots+\cdots+ \pm \pm) & \text { für } \beta=2 n+1
\end{array}
$$

und im Falle

$$
\begin{aligned}
& r=(\cdots+\cdots+ \pm \pm), \quad \tilde{r}=(\cdots+\cdots- \pm \pm) \\
& \varrho=(\cdots+\cdots+ \pm \pm) \quad \text { für } \beta=2 m+1 \\
& \tilde{\varrho}=(\cdots-\cdots- \pm \pm) \quad \text { für } \beta=2 m .
\end{aligned}
$$

wie nach der Regel von Nr. 4 bestätigt werden kann.

Unsere Behauptung ist damit vollständig bewiesen.

Nach dem Obigen kann man auf (irund unserer Regel die Gesamtheit der reellen Nullstellen eines Polynoms $G_{n}(p)$ dunch eine Zeichenfolge $(4,2)$ darstellen, sobald man das erste Glied der Folge lennt. welche der kleinsten Nullstelle des Polynoms entspricht. Für die kleinsten Werte ron $n$ bestehen die betrefferden minimalen Radikale aus den Folgenden, wie leicht zu bestätigen ist:

$$
\begin{aligned}
& n=3:(+), \quad n=4:(+-), n=5:(+--), n=6:(+---), \\
& n=7:(+----), \quad n=8:(+---+-), \\
& n=9:(+-\ldots----) ; n=10:(+---+-+-) .
\end{aligned}
$$

Die Folgen selbst haben wir im Anhang in einer Tafel zusammengestellt. 


\section{Die Anzahl der reellen Nullstellen der $G$-Polynome.}

6. Wir sind jetzt in der Lage einen expliziten Ausdruck für die Anzahl der reellen Nullstellen der G-Polynome zu geben.

Es seien

$$
\lambda_{1}, \lambda_{2}, \ldots, \lambda_{N}
$$

die Fixpunkte von $(1,2)$, also die Wurzeln der Gleichung

$$
y_{n}-y=0 .
$$

Man hat

$$
\prod_{v=1}^{N} \lambda_{v}=G_{n}(p)=p_{n-1}, \quad \prod_{v=1}^{N} \lambda_{v} \cdot \sum_{\mu=1}^{N} \frac{1}{\lambda_{\mu}}=1 .
$$

Es sei nun $G_{n}\left(p_{0}\right)=0$ und z.B. $\lambda_{1}=0$. Aus der zweiten Gleichung $(6,3)$ ergibt sich $\prod_{r=2}^{N} \lambda_{v}=1$. Für $p \approx p_{0}$ ist somit, wegen der ersten Gleichung, $\lambda_{1} \approx p_{n-1}$. Für den Multiplikator des zum Fixpunkt $\lambda_{1}$ gehörigen Zyklus $\sum$ ergibt sich hieraus für $p \approx p_{0}$ der approximative Ausdruck

$$
S \approx-2^{n} p p_{1} p_{2} \ldots p_{n-1}
$$

Es sei nun $p_{0}$ die kleinste reelle Nullstelle von $G_{n}(p)$, für welche $\lambda_{1}=0$ wird. Sei ferner $\tilde{p}_{0}$ derjenige Wert des Parameters $p$, für welchen der Zyklus $\Sigma$ reell wird. Es ist dann $S=+1$ für $p=\tilde{p}_{0}$ und $S>0$ im Intervall $\Delta_{0}\left(\tilde{p}_{0}, p_{0}\right)$. Weil die Nullstellen von $G_{n}$ zufolge unserer Hypothese $H$ einfach sind, muss $S<0$ gelten im Intervall $\left(p_{0}, p_{0}{ }^{\prime}\right)$, wo $p_{0}$ die nächste Nullstelle von $G_{n}$ bezeichnet.

Nun ist aber die Zeichenfolge der Grössen

$$
p, p_{1}, p_{2}, \ldots, p_{n-1}
$$

in einem Nullpunkt von $G_{n}$ gleich der Zeichenfolge in dem zugehörigen Radikal. Hieraus folgt, nach Nr. 5, der

Hilfssatz. Beim Übergang einer Nullstelle ron $G_{n}$ geht das Vorzeichen des Produktes

$$
-p p_{1} p_{2} \cdots p_{n-1}
$$

stets bei wachsendem $p$ von + in - über.

Weil nach dem U̇bergang der grössten Nullstelle das Produkt $(6,6)$ sein - Zeichen behält und weil für alle reellen Nullstellen $p<2$ ist, gilt der 
Satz 2. Die Anzahl der reellen Nullstellen des Polynoms $G_{n}(p)$ ist gleich der Anzahl der negativen Zyklen von $y_{n}$ für $p=2$, d.h. Zyklen mit einem negativen Multiplikator.

Unser Problem wird dadurch auf die Bestimmung der Zyklen von $y_{n}$ für den Wert $p=2$ zurückgeführt. Nach [4] bilden die Fixpunkte von $y_{n}$ für $p=2$ zwei Gruppen

$$
\begin{array}{ll}
\text { I : } \quad 2 \cos \frac{2 \pi m}{2^{n}-1}, \quad\left(m=0,1, \ldots, 2^{n-1}-1\right) \\
\text { II : } \quad 2 \cos \frac{2 \pi m^{\prime}}{2^{n}+1}, \quad\left(m^{\prime}=1,2, \ldots, 2^{n-1}\right) .
\end{array}
$$

Ist

$$
\cos 2^{v} \alpha_{m}, \quad(v=1,2, \ldots, n-1)
$$

ein Zyklus der ersten Gruppe, so ist sein Multiplikator

$$
S=2^{n} \prod_{\nu=0}^{n-1} \cos 2^{\nu} \alpha_{m}=2^{n} \sin \frac{2 \pi m}{2^{n}-1}>0 .
$$

Für die Zyklen der zweiten Gruppe gilt dagegen

$$
S=-2^{n} \sin \frac{2 \pi m^{\prime}}{2^{n}+1}<0 .
$$

Indem wir nur die primitiven Nullstellen von $G_{n}$ berücksichtigen, können wir aus dem Obigen den folgenden Satz ableiten.

Satz 3. Die Anzahl der primitiven Nullstellen des Polynoms $G_{n}$ ist gleich der Anzahl der primitiven Zyklen in der Gruppe II.

Für diese Anzahl gilt der Ausdruck

$$
\varphi(n)=\frac{2^{n-1}-\varepsilon_{n}}{n} .
$$

Hier ist $\varepsilon_{n}=0$ für $n=2^{m}$ und $\varepsilon_{n}=1$ für eine ungerade Primzahl $n$. Allgemein ist

$$
\varepsilon_{n}=n \sum_{\nu} \frac{\varphi\left(\frac{n}{k_{v}}\right)}{k_{v}},
$$

wo $k_{v}$ die Gesamtheit der ungeraden Teiler von $n$ zu durchlaufen hat.

Für die kleinsten Werte von $n$ bekommt man den zugehörigen Wert von $\varepsilon_{n}$ und $\varphi(n)$ aus der folgenden Tafel 


\begin{tabular}{|c|r|r|r|r|r|r|r|r|r|r|r|r|r|r|r|}
\hline$n$ & 2 & 3 & 4 & 5 & 6 & 7 & 8 & 9 & 10 & 11 & 12 & 13 & 14 & 15 & 16 \\
\hline$\varepsilon_{n}$ & 0 & 1 & 0 & 1 & 2 & 1 & 0 & 4 & 2 & 1 & 8 & 1 & 2 & 19 & 0 \\
\hline$\varphi(n)$ & 1 & 1 & 2 & 3 & 5 & 9 & 16 & 28 & 51 & 93 & 170 & 315 & 585 & 1091 & 2048 \\
\hline
\end{tabular}

Die Wurzelausdrücke und die approximativen Zahlenwerte der reellen primitiven Nullstellen der 10 ersten Polynome $G_{n}$ sind am Schlusse dieser Abhandlung gegeben.

\section{Gegenseitige Lage der Nullpunkte der $G$-Polynome.}

7. Nach dem Obigen bilden die reellen Nullpunkte $\neq 0$ der $G$-Polynome eine unendliche, im Intervall $1 \leqq p<2$ liegende Punktmenge. Wir behaupten, dass die genannten Punkte isoliert liegen. Wäre nämlich ein solcher Punkt $p_{0}$ eine Häufungsstelle für andere Nullpunkte, so könnte man um $p_{0}$ herum ein Intervall bilden, für dessen $p$-Werte mehrere, ja sogar unendlich viele verschiedene attraktive Zyklen vorhanden wären, was dem Fatouschen Satz widerspricht.

Es sei nun $\alpha_{n}$ ein Nullpunkt von $G_{n}$ mit der Entwicklung

$$
p=r_{n}(p) \text {. }
$$

Wir bilden den Ausdruck

$$
r_{n+1}=r_{n}( \pm-) r_{n}
$$

wo das Doppelzeichen + oder - ist, je nachdem ob die Anzahl der Zeichen in $r_{n}$ gerade oder ungerade ist. Aus den Entwicklungen von Nr. 4 geht hervor, dass

$$
p=r_{n+1}(p)
$$

einen Nullpunkt $\alpha_{2 n}>\alpha_{n}$ von $G_{2 n}$ darstellt und dass zwischen den Punkten $\alpha_{n}, \alpha_{2 n}$ kein Nullpunkt eines G-Polynoms existiert.

Aus dem Obigen folgt, dass man von einem Nullpunkt $\alpha_{n}$ von $G_{n}$ ausgehend eine monoton wachsende Folge

$$
\alpha_{n}<\alpha_{2 n}<\alpha_{4 n}<\ldots
$$

von Nullpunkten der Polynome

$$
G_{2^{m_{n}}} \quad(m=0,1,2,3, \ldots)
$$

bilden kann, so dass zwischen zwei Punkten von $(7,4)$ keine anderen Nullpunkte der G-Polynome existieren. Wir können offenbar ohne Einschränkung annehmen, dass die Folge $(7,4)$ nicht rückwärts fortgesetzt werden kann. Wir wissen ferner nach dem Obigen, dass für $(7,4)$ der Grenzwert 


$$
\lim _{m \rightarrow \infty} \alpha_{2^{m} n}=\alpha^{*}
$$

existiert, der jedenfalls $<2$ ist.

Wir bilden jetzt den Ausdruck

$$
r_{n+1}(p)=\sqrt{p-r_{n}(p)} .
$$

welcher für $p \geqq \alpha_{n}$ reell ist und für $p=\alpha_{n}$ verschwindet. Der durch $(7,6)$ definierte Punkt gehört zur Menge $\mu$ der aus $y=0$ durch Iteration der zu der inversen Operation $\sqrt{y+p}$ erhaltenen Bildpunkte. Wir behaupten nun, dass das Intervall

$\delta:$

$$
0<y<r_{n+1}(p)
$$

keinen Punkt der Menge $\mu$ enthält.

Wir nehmen zum Beweis indirekt an, es sei $r(p)$ ein Punkt von $\mu$, der für einen Wert $p$ des Intervalles $\delta$ zu $\mu$ gehört, also

$$
0<r(p)<r_{n+1}(p) .
$$

Weil $\alpha_{n}$ eine primitive Nullstelle von $G_{n}$ ist, gilt $r\left(\alpha_{n}\right)>0$. Es sind jetzt a priori zwei Fälle möglich: 1. Für einen Wert $p_{0}$ von $\delta$ ist $r(p)=r_{n+1}(p)$. 2. Beim Übergang über einen Wert $p_{0}$ von $\delta$ wird $r(p)$ imaginär und also $r\left(p_{0}\right)=0$. In den beiden Fällen wird aber offenbar ein Teilradikal von $r(p)$ gleich null, und dort hätte ein Polynom $G(p)$ einen Nullpunkt. was dem Obigen widerspricht. Unsere Behauptung ist damit bewiesen.

Gleiches gilt offenbar für jedes Intervall $\Delta_{m}=\left(\alpha_{2^{m}}, \alpha_{2^{m+1}}\right)$.

\section{Existenz von attraktiven Zyklen}

8. Wir sind jetzt in der Lage, eine allgemeine Nethode zur Konstrultion von attraktiven Zyklen zu geben.

Aus den Entwicklungen der Nr. 6 wissen wir schon, dass um jeden Punkt $\alpha_{2} m_{n}$ ein $p$-Intervall konstruiert werden kann, für dessen Werte ein attraktiver Zyklus der Ordnung $2^{m} n$ existiert, der für $p<\alpha_{2^{m}}$ positiv und für $p>\alpha_{2} m_{n}$ negativ ist und für $p=\alpha_{2^{m_{n}}}$ in einen Nullzyklus übergeht. Speziell für den ersten Punkt gilt dabei folgendes: es gibt ein Intervall

$$
\alpha_{0}<p<\alpha_{n}
$$

für dessen $p$-Werte ein positiver attraktiver Zyklus der Ordnung $n$ existiert, der für $p=\alpha_{0}$ indifferent wird. Die Punkte

$$
\alpha_{0}<p<\alpha^{*}
$$


definieren dann ein Intervall $\sigma_{n}$ von $p$-Werten, das wir ein Spektrum nennen werden. Die Bedeutung des Spektrums geht nun aus dem folgenden Satz hervor,

Satz 4. Für jeden zum Spektrum $\sigma_{n}$ gehörigen $p$-Wert gibt es einen attraktiven Zyklus, dessen Ordnung in einem Teilintervall

$\Delta_{m}$ :

$$
\left(\alpha_{2^{m}}, \alpha_{2} m+1_{n}\right)
$$

gleich $2^{m} n$ oder $2^{m+1} n$ ist.

Wir bemerken zum Beweis am Anfang, dass der Wert der Ableitung

$$
y_{m}^{\prime}=2^{m} y y_{1} \ldots y_{m-1}
$$

für jede Iterierte $y_{m}$ auf $\delta=\delta(0, r)$ von null verschieden ist, weil dort kein Punkt von $\mu$ liegt. Vermittels $y_{m}$ wird also das Intervall $\delta(0, r)$ schlicht auf ein Intervall $\delta_{m}\left(p_{m-1}, r_{m}\right)$ abgebildet, das wir kurz durch

$$
y(0, r) \rightarrow y_{m}\left(p_{m-1}, r_{m}\right)^{ \pm}
$$

ausdrücken, wo das Zeichen + oder - ist, je nachdem ob das Intervall $\delta_{m}$ positiv oder negativ gerichtet ist. Weil ferner $\delta_{m}$ den Nullpunkt nicht enthält, ist allgemein

$$
\operatorname{sign} p_{m-1}=\operatorname{sign} r_{m}
$$

und speziell

$$
\operatorname{sign} p_{v+n}=\operatorname{sign} p_{v} .
$$

Nun ergibt sich aus $(8,3)$ alternierend

$$
y(0, r) \rightarrow y_{n+1}\left(p_{n}, p\right)^{-} \rightarrow y_{2 n+1}\left(p_{2 n}, p_{n}\right)^{+} \rightarrow \cdots,
$$

also

wobei

$$
\begin{gathered}
-p<p_{2 n}<p_{4 n}<\cdots, \\
0>p_{n}>p_{3 n}>p_{5 n}>\cdots
\end{gathered}
$$

Hieraus folgt ferner

$$
p_{(2 k-1) n}>p_{2 k n}
$$

$$
\begin{aligned}
& y\left(-p, p_{2 n}\right)^{+} \rightarrow y_{2 n}\left(p_{2 n}, p_{4 n}\right)^{+} \rightarrow y_{4 n}\left(p_{4 n}, p_{6 n}\right)^{+} \rightarrow \cdots \\
& y\left(p_{n}, p_{3 n}\right)^{-} \rightarrow y_{2 n}\left(p_{3 n}, p_{5 n}\right)^{-} \rightarrow y_{4 n}\left(p_{5 n}, p_{7 n}\right)^{-} \rightarrow \cdots
\end{aligned}
$$

Es existieren somit die Grenzwerte

$$
\begin{aligned}
& \lim _{k \rightarrow \infty} y_{2 k}\left(p_{2 k}, p_{2 k+2}\right)=\lambda \\
& \lim _{k \rightarrow \infty} y_{2 k+1}\left(p_{2 k+1}, p_{2 k+3}\right)=\lambda^{\prime},
\end{aligned}
$$


wo $\lambda \leqq \lambda^{\prime}$. Diese Grenzwerte geben offenbar Fixpunkte für einen attraktiven (im speziellen Falle indifferenten) Zyklus, dessen Ordnung gleich $2 n$ im Falle $\lambda<\lambda^{\prime}$ und gleich $n$ im Falle $\lambda=\lambda^{\prime}$ ist. Weil gleiches für jedes Intervall $\Delta_{m}$ gilt, ist unser Satz 4 damit endgültig bewiesen.

Wir bemerken noch, dass die Ordnung des zum Intervall $\Delta_{m}$ gehörigen Zyklus in der Nähe des Wertes $p=\alpha_{2^{m}}$ gleich $2^{m} n$ und in der Nähe des Wertes $p=\alpha_{2^{m+1}}$ gleich $2^{m+1} n$ ist und daş der Übergang für Werte von $p$ stattfindet, wo ein indifferenter Zyklus mit dem Multiplikator $S=-1$ vorliegt.

Als Beispiel wählen wir das seiner Lage nach extreme Spektrum $\sigma_{0}$ mit dem Anfangspunkt $p=1 / 4$. Die zugehörigen Punkte $(7,4)$ sind jetzt die kleinsten Nullpunkte der Polynome

$$
G_{2 m} \quad(m=1,2,3, \ldots),
$$

und sie sind Wurzeln der Gleichungen

$$
p=r_{m}
$$

wo die Radikale $r_{m}$ aus der Rekursionsformel

$$
r_{m+1}=r_{m}( \pm-) r_{m}
$$

berechnet werden können. Hier gilt das + Zeichen für ungerades $m$ und - Zeichen für gerades $m$. Die ersten unter den Grössen $\alpha_{m}$ haben die folgenden approximativen Werte $\left(\alpha_{1}=1\right)$

$$
\begin{gathered}
\alpha_{2}=(+-)=1,3107026413 \\
\alpha_{3}=(+--+-)=1,3815474844 .
\end{gathered}
$$

Für den Grenzwert $\lim _{m \rightarrow \infty} \alpha_{m}=p_{0}^{*}$, den Endpunkt des Spektrums $\sigma_{0}$, habe ich vermittels einer Elektronenmaschine den approximativen Wert

berechnet.

$$
p_{0}^{*}=1,401155189
$$

\section{Singuläre $p$-Werte}

9. Wir nennen einen $p$-Wert $p^{*}$ singulär, wenn der Punkt $p=p^{*}$ eine Häufungsstelle der Nullstellen der G-Polynome ist. Nach dem Obigen gehören zur Menge $\left(p^{*}\right)$ der singulären $p$-Werte u.a. die Endpunkte der Spektren. Eine andere allgemeine Methode zur Bildung singulärer $p$-Werte findet man in unserer Arbeit [5]. Wegen der Definition ist $\left(p^{*}\right)$ eine unendliche abgeschlossene Menge, welche $p=p_{0}^{*}$ als kleinstes und $p=2$ als grösstes Element enthält. Eine bemerkenswerte Art von singulären Werten ergibt sich aus dem folgenden 
Satz 5. Der Anfangspunkt jedes Spektrums ist ebenfalls singulär, vom Anfangspunkt $p=1 / 4$ des ersten Spektrums $\sigma_{0}$ abgesehen.

Es sei nämlich $\sigma_{n}$ ein Spektrum der Ordnung $n$ mit dem Anfangspunkt $\alpha_{n}^{0}$ und der ersten Nullstelle $\alpha_{n}$. Für die $p$-Werte des Intervalles $\left(\alpha_{n}^{0}, \alpha_{n}\right)$ gelten dann die Beziehungen

$$
y\left(-p, p_{n}\right)^{+} \rightarrow y_{n}\left(p_{n}, p_{2 n}\right)^{+} \rightarrow y_{2 n}\left(p_{2 n}, p_{3 n}\right)^{+} \rightarrow \cdots
$$

wobei der Grenzwert $\lim p_{v_{n}}$ einen attraktiven Fixpunkt des entsprechenden Zyklus $\Sigma_{n}$ gibt. Die Relationen $(9,1)$ können aber nicht mehr beim Übergang des Wertes $\alpha_{n}^{0}$ gelten, weil der Zyklus $\Sigma_{n}$ jetzt nicht mehr attraktiv ist. Hieraus folgt, wie leicht einzusehen, dass gewisse Punkte $p_{v}$ den Nullpunkt überschreiten müssen. Weil solche Punkte, also Nullstellen der $G$-Polynome, in jeder Umgebung des Wertes $\alpha_{n}^{0}$ existieren, ist unser Satz damit bewiesen.

10. Um das Obige näher an einem Beispiel zu erläutern, betrachten wir das zu $G_{3}$ gehörige Spektrum mit dem Anfangspunkt $p=7 / 4$. Wir bilden jetzt die unendliche Folge der Radikalgleichungen

$$
p=(+--)^{m}=r_{m}, \quad(m=1,2,3, \ldots)
$$

welche Nullstellen der Polynome

$$
G_{2+3 m}
$$

geben. Man bestätigt nach der in Nr. 5 angegebenen Methode, dass die genannten Nullstellen $\omega_{m}$ eine monoton wachsende Folge bilden:

\section{Der Grenzwert}

$$
\omega_{1}<\omega_{2}<\omega_{3}<\cdots
$$

$$
\lim _{v \rightarrow \infty} \omega_{v}=\omega^{*}
$$

genügt dann, wegen des Fatouschen Satzes, der Bedingung $\omega^{*} \leqq 7 / 4$.

Wir behaupten nun, dass $\omega^{*}=7 / 4$. Andernfalls wären alle Radikale $r_{m}$ und Grössen $\omega_{m}$ reell für einen Wert $p<7 / 4$, und dies wäre auch mit dem Grenzwert $\omega^{*}$ der Fall. Das ist aber unmöglich. Denn offenbar genügt $x=\omega^{*}$ der Gleichung

also der Gleichung

$$
x=\sqrt{p+\sqrt{p-\sqrt{p-x}}},
$$

$$
\left[\left(x^{2}-p\right)^{2}-p\right]^{2}-p=-x,
$$

woraus folgen würde, dass $-x$ ein reeller Fixpunkt von $y_{3}$ wäre, was ein Widerspruch ist [4]. 
Nach dem Obigen hat der Anfangspunkt jedes von $\sigma_{0}$ verschiedenen Spektrums die Eigenschaft, einseitig singulär zu sein, wobei für den Anfangswert ein indifferenter Zyklus mit dem Multiplikator $S=+1$ existiert. Jeder solcher Punkt ist gleichzeitig Häufungspunkt für Spektren.

Was die Endpunkte der Spektren betrifft, ist es wahrscheinlich, dass sie beiderseits singulär sind. Ohne unsere Vermutung beweisen zu können, wollen wir zu ihrer Begründung folgendes bemerken.

Wir betrachten den Endpunkt $p_{0}^{*}$ des ersten Spektrums, der nach Nr. 8 von links eine Häufungsstelle der kleinsten Nullstellen der Polynome $G_{2^{n}}$ ist. Es sei $p_{1}^{*}$ die untere Grenze der rechts von $p_{0}^{*}$ liegenden Nullstellen der G-Polynome, wobei wegen des Fatouschen Satzes $p_{1}^{*} \geqq p_{0}^{*}$ gilt. Unsere Hypothese ist hier äquivalent mit der Gleichung

$$
p_{1}^{*}=p_{0}^{*} \text {. }
$$

Zur praktischen Verifizierung dieser Gleichung haben wir vermittels einer Elektronenmaschine die kleinsten reellen Nullstellen aller G-Polynome $G_{m}$ bis zum Wert $m=29$ berechnet und dabei bestätigt, dass

$$
p_{1}^{*}<1,40117,
$$

wo rechts eine Grösse vorkommt, die sich von $p_{0}^{*}$ um weniger als $2.10^{-5}$ unterscheidet. Gleichzeitig erhält man hieraus für den Wert von $p_{0}^{*}$ die Schranken

$$
1,40115<p_{0}^{*}=1,40117 .
$$

Weil ähnliches für alle Spektren gilt, folgt aus unserer Hypothese der

Satz 5. Die Summe der Spektren überdeckt das Intervall

$$
1 / 4 \leqq p \leqq 2
$$

bis auf eine unendliche diskontinuierliche Punktmenge $\left(p^{*}\right)$. die Menge der singulären Werte des Parameters $p$.

Für jeden zu $\left(p^{*}\right)$ nicht gehörigen Wert ron $p$ existiert stets ein attraktiver Zyklus, dessen Ordnung bei unbegrenzter Annäherung an einen Punkt von $\left(p^{*}\right)$, wenigstens von einer Seite, über alle Grenzen wächst.

\section{Nachtrag}

Wenn man die Hypothese $H$ nicht annehmen will, hat man in den Sätzen 2-4 folgende Modifikationen zu machen.

Sätze 2 und 3. Die Anzahl der primitiven Nullstellen des Polynoms $G_{n}$ ist wenigstens gleich $\varphi(n)$.

Satz 4. Gilt mit dem Zusatz, dass für gewisse Teilintervalle der Spektren attraktive Zyklen vorkommen können, die aus Nullzyklen nicht durch stetige Variation des Parameters abgeleitet werden können. 
Tafel für die approximativen Werte der reellen Nullstellen der $G$-Polynome

$G_{3}=0$ :

$1,7548776662=(+)$

$G_{1}=0:$

$1,3107026413=(+-)$

$1,9407998065=(++)$

$G_{5}=0:$

$1,6254137251=(+--)$

$1,8607825222=(++-)$

$1,9854242531=(+++)$

$G_{6}=0:$

$1,4760146427=(+---)$

$1,7548776662=(+--+)=(+)$

$1,7728929034=(++-+)$

$1,9072800911=(++--)$

$1,9667732164=(+++--)$

$1,9963761377=(++++)$

$G_{7}=0:$

$1,5748891398=(+-\ldots--)$

$1,6740660915=(+--+-)$

$1,8323152028=(++-+-)$

$1,8848035716=(++---)$

$1,9271477094=(++-\cdots+)$

$1,9537058943=(+++-+)$

$1,9771795870=(+++--)$

$1,9918141725=(++++-)$

$1,9990956823=(+++++)$

$G_{8}=0$ :

$1,3107026413=(+-+-+-)=(+-)$

$1,3815474844=(+\cdots-+-)$

$1,0218172317=(+-\ldots--)$

$1,7110794700=(+-\ldots+--)$

$1,8100013858=(++-+--)$

$1,8517300494=(++-+--)$

$1,8700038808=(++----)$

$1,8969179947=(++----)$

$1,9170982771=(++--+-)$

$1,9407998065=(++--++)=(++)$

$1,9417820903=(+++-++)$

$1,9607589872=(+++-+-)$

$1,9721998384=(+++---)$

$1,9816557863=(+++--+)$

$1,9887932743=(++++-+)$

$1,9943329667=(++++--)$
$G_{8}=0:$

$1,9979629156=(+++++-)$

$1,9997740487=(+++++-)$

$G_{9}=0$ :

$1,5552827008=(+\cdots-\cdots+\cdots)$

1.5956809634 $=(+-\ldots+\ldots-)$

$1,6561325626=(+-++-\cdots)$

$1,6901422631=(+\cdots++--)$

$1,7548776662=(+--+-+)=(+)$

$1,7858656464=(++-+-++)$

$1,8227563225=(++\cdots+\cdots-)$

$1,8412885615=(++-+---)$

$1,8783826015=(++\ldots-+-)$

$1,8907754244=(++\ldots-\ldots-)$

$1,9031167730=(++--\cdots+)$

$1,9114446315=(++-+++)$

$1,9222857782=(++\ldots+\cdots)$

$1,9322439666=(++-++-\infty)$

$1,9495749032=(+++-++-)$

$1,9573250505=(+++-+--)$

$1,9640243368=(+++-++)$

$1,9694191205=(+++-\ldots+)$

$1,9747808589=(+++-\ldots-)$

$1,9794575049=(+++-+--)$

$1,9838102500=(+++--++)$

$1,9870043475=(++++-++)$

$1,9903763811=(++++-+-)$

$1,9931302549=(++++---)$

$1,9954190327=(++++--+)$

$1,9972230247=(+++++-+)$

$1,9985865888=(+++++--)$

$1,9994914380=(++++++-)$

$1,9999435218=(+++++++)$

$G_{10}=0$ :

$1,4470088406=(+---+-+-)$

$1,5017168394=(+-\ldots--+-)$

$1,5362432713=(+-\ldots-\ldots--)$

$1,6254137251=(+-\ldots-+--)=(+--$

$1,6294325004=(+--+-+--)$

$1,7017008912=(+--+---)$

$1,7219150996=(+--+--+-)$

$1,8024361348=(++-+--+-)$

$1,8162948552=(++-+----)$

$1,8295096240=(++-+--+)$

$1,8351588453=(++-+-+-+)$

$1,8466272112=(++-+-+-)$

$1,8607825222=(++-+++-)=(++-$ 
$G_{10}=0:$

\begin{tabular}{|c|c|c|}
\hline $1,8615584138=(++-$ & -- & ++-1 \\
\hline $1,8743146838=(++-$ & -- & $+--)$ \\
\hline, $8824079338=(++-$ & -- & +-+1 \\
\hline $8871722064=(++-$ & - - & $--+)$ \\
\hline $8940020229=(++-$ & -- & ++1 \\
\hline $1,8998323378=(++-$ & -+ & ++1 \\
\hline $1,9144798637=(++$ & -+ & $+-)$ \\
\hline $1,9196354893=(++-$ & -+ & $---)$ \\
\hline $1,9250342385=(++-$ & -+ & --+1 \\
\hline $1,9293203309=(++-$ & -+ & +-+1 \\
\hline $1,9353907348=(++-$ & -+ & +--1 \\
\hline $1,9468734542=(+++$ & -+ & +--1 \\
\hline $1,9518999969=(+++$ & -+ & $+-+)$ \\
\hline $1,9554233270=(+++$ & -+ & $---+)$ \\
\hline $1,9590980466=(+++$ & -+ & ---1 \\
\hline $1,9623786897=(+++$ & -+ & +-1 \\
\hline $1,9658217855=(+++$ & -+ & $-++)$ \\
\hline $1,9677427763=(+++$ & -- & $-++)$ \\
\hline $1,9708578891=(+++$ & -- & $-+-)$ \\
\hline $1,9734974173=(+++$ & -- & ---1 \\
\hline $1,9760421686=(+++$ & - - & --+1 \\
\hline
\end{tabular}

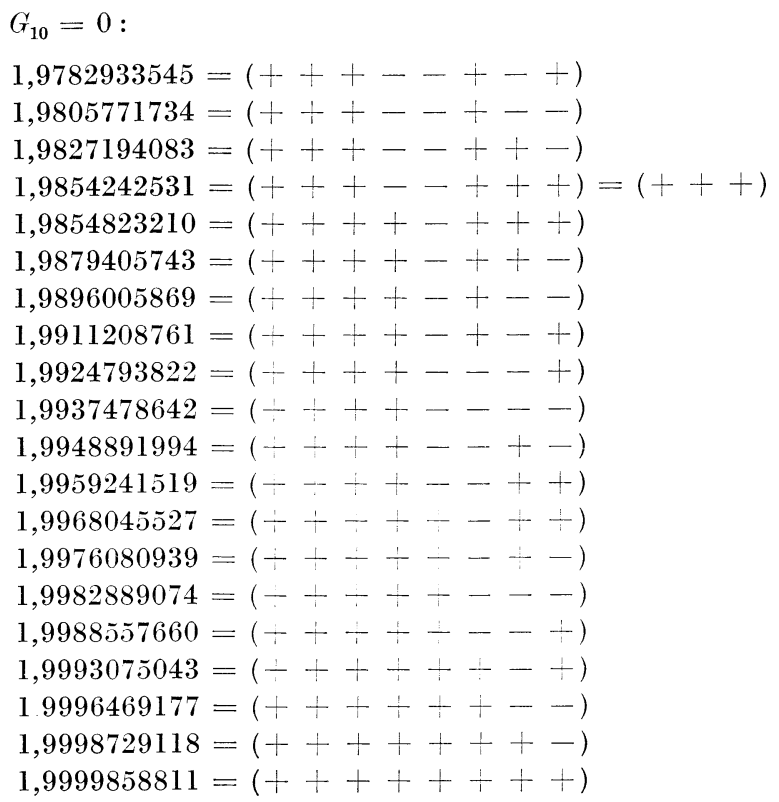

\section{Verzeichnis der früheren Arbeiten des Verfassers über die Iteration}

[1] Sur une généralisation de la moyenne arithmétique-géométrique de Gauss (C. R. Acad. Sc., t. 246, 1958).

[2] Eine Verallgemeinerung des arithmetisch-geometrischen Mittels (Ann. Acad. Sc. Fennicae, A. I, no. 253, 1958).

[3] Iteration der reellen Polynome zweiten Grades (Ibid. no. 256, 1958).

[4] Iteration von Quadratwurzeloperationen (Ibid, no. 259, 1958).

[5] Iteration der reellen Polynome zweiten Grades. II. (Ibid. no. 268, 1959).

[6] Funktioista, jotka toteuttavat toisen asteen kertosäännön. (Über Funktionen, die ein quadratisches Multiplikationstheorem besitzen) (Arkhimedes no. 1, 1960).

[7] Inversion der Iteration für rationale Funktionen (Ann. Acad. Sc. Fennicae, A. I., no. 292, 1960).

[8] Funktioista, jotka toteuttavat toisen asteen kertosäännön (Arkhimedes no. 1, 1962)

[9] Sur l'itération des polynomes réels quadratiques (Journal de Math. 1962). 